\section{CHARACTERISATION OF A Zn TRIFLATE-BASED POLYMER ELECTROLYTE}

M. Manuela Silva a, M. J. Smith *, a, P. Lightfoot b

a Centro de Quimica, Universidade do Minho, Gualtar, 4710 Braga, Portugal

b Department of Chemistry, University of St. Andrews, St. Andrews, $U K$

Abstract

Ionic conductivity measurements, $\mathrm{X}$-ray diffraction analysis and thermal studies have been carried out on polymer electrolyte films prepared by the addition of zinc trifluoromethanesulphonate (triflate) to poly(ethylene oxide), PEO. In general this electrolyte system was found to behave in a manner similar to other divalent salt-based electrolytes with an increase in crystalline morphology as the concentration of the salt component in the electrolyte was decreased. The conductivity isotherms calculated for this system show an almost constant level of ionic conductivity over the entire range of composition studied.

Keywords - polymer electrolyte, poly(ethylene oxide), thermal stability, ionic conductivity

\section{Introduction}

Since 1978, when poly(ethylene oxide) based electrolytes were first presented as potential battery components [1], the principle motivation for the study of these materials has been provided by the promise of commercial applications in high energy density primary and secondary batteries. Shortly after the introduction of the solid polymer electrolyte concept, other structurally related host polymers combined with a remarkable variety of di- and trivalent guest species were studied [2-4]. The characterisation of these novel electrolytes led to the conclusion that their behaviour is determined not only by the choice of host polymer and the nature of the guest ionic species, but also by the preparative conditions used and even the thermal history of the sample.

While lithium salt-based systems offer significant practical advantages derived from the high electrochemical potential and low density of the active element, problems associated with the stability of the electrode/electrolyte interface have delayed commercialisation of advanced batteries based on solid polymer electrolytes. Several authors have suggested that zinc, a well-characterised material used in conventional aqueous systems, might be a convenient alternative anode for polymer battery systems and various polymer electrolytes based on different zinc salts have been already been described $[3,5-12]$. In this study a wide range of electrolyte compositions, formed by dissolution of zinc triflate in commercial PEO, was characterised by total ionic conductivity measurements, structural techniques and thermal analysis.

Almost all polymer electrolytes based on PEO are semicrystalline materials with complex microstructures. Within certain composition ranges these polymer samples include spherically symmetrical arrangements of lamellar fibrils which radiate from a central nucleus. The growth of these spherulites results from the alignment of macromolecules on the surfaces of fibrils which branch to fill a high fraction of the inter-fibril volume. The extent of crystallisation in this polymer environment depends not only on the chemical nature and composition of the system, but also on the precise experimental conditions (choice and purity of casting solvent, concentration of the casting solution, thermal history of the sample, etc). Amorphous material is always present in the regions between fibrils and in the inter-spherulitic domains. As the rate of formation of the crystalline phases present in polymer electrolytes depends on the diffusion of polymer chains through a viscous environment, the kinetics of crystalline phase formation are slow and pseudoequilibrium phase diagrams are often used to provide a convenient descriptive model which can be helpful in interpreting the observed conductivity and mechanical properties of the system.

The presence of the guest salt in the host polymer alters the behavior of the polymer to an extent which depends on the nature and quantity of the species present. In most electrolytes containing PEO at low concentrations of added salt, the guest species is expelled from the polyme spherulites into the amorphous inter-spherulitic region [13]. Normally a range of composition may be identified in which the crystallinity of the electrolyte decreases as the amount of added salt increases (or $\mathrm{n}$ decreases). The kinetics of growth of polymer spherulites become slower as the quantity of salt in the electrolyte increases. In some electrolyte systems crystallisation from melted samples is so slow that marked differences between the conductivity data obtained during the heating and cooling cycles are observed [14]. This behaviour is frequently observed at salt contents close to the system eutectic composition.

In what is normally referred to as the salt-rich region of composition, the presence of salt results in the formation of an almost completely amorphous material. This behaviour is considered to be a result of salt-polymer interactions which impede the motion, long and short-range, of the polymer segments. These physical restrictions also cause a significant reduction in the ionic conductivity as the mechanism of ion transport is dependent on the polymer chain mobility. Some systems form crystalline complexes between the guest salt and host polymer in this composition region and again this causes a dramatic reduction in conductivity as the number of mobile ions available for charge transport is reduced. The existence of these salt-polymer complexes in some systems has been demonstrated by structural and thermal techniques $[15,16]$. At even higher salt concentrations the system reaches a point beyond which further solubilisation of salt is not possible and salt deposition or phase separation occurs.

\section{Experimental}

In recognition of the effect of water on polymer electrolyte behaviour [17], zinc triflate (Aldrich, 98\%) and PEO (Aldrich, Mw $5 \times 10^{6}$ ) were dried under vacuum for 7 days at 200) an $55^{\circ} \mathrm{C}$, respectively. All subsequent operations involving the manipulation of electrolyte components, the preparation of samples for conductivity measurements, thermal and X-ray analysis, were carried out under a dry argon atmosphere within high integrity gloveboxes. Films of electrolyte were prepared by dissolving PEO and zinc triflate in acetonitrile (Aldrich, HPLC grade) which had been previously dried over regenerated molecular sieves (Aldrich, 4A). The composition of the electrolyte produced was determined by the masses of polymer and salt dissolved in the casting solvent and designated by $\mathrm{n}$, the ratio of polymer repeat units to salt ion group ( $\mathrm{n}$ is alse therefore the ratio $\mathrm{O} / \mathrm{Zn}^{2+}$ ). After prolonged stirring the homogeneous solution of polymer and salt 
was transferred to a teflon mould and the solvent was evaporated by passing a dry argon flow over the polymer solutions. This process took place in a closed compartment within a preparative argonfilled glovebox. The mould supporting the resulting polymer film was transferred to a Buchi TO51 tube oven and subjected to a final drying process, at $50^{\circ} \mathrm{C}$ for 72 hours under vacuum, to remove solvent residues.

The total ionic conductivity of samples was determined by locating an electrolyte disk between two $10 \mathrm{~mm}$ diameter ion-blocking gold electrodes (Goodfellow, > 99.9\%). The electrolyte/electrode assembly was secured in a suitable constant volume support [18] which allowed extremely reproducible measurements of conductivity to be obtained between repeated heating/cooling cycles. The cell support was located in a Buchi TO51 tube oven and the sample temperature was measured by a type $\mathrm{K}$ thermocouple positioned close to the electrolyte disk. The bulk conductivities of electrolyte samples were obtained during a heating cycle using the complex plane impedance technique (Schlumberger Solartron 1250 frequency response analyser and 1286 electrochemical interface) over a temperature range of between 25 and $100{ }^{\circ} \mathrm{C}$ and at approximately $7^{\circ} \mathrm{C}$ intervals.

Open platinum cans were used to support the electrolyte samples during thermogravimetric analysis (TGA). The electrolyte samples, in the form of $5 \mathrm{~mm}$ diameter disks, were cut from electrolyte films within the glovebox. The samples were transferred to the cans and located within the thermobalance with the shortest possible exposure to the laboratory atmosphere. Analysis took place under a flowing nitrogen atmosphere $\left(30 \mathrm{~cm}^{3} \mathrm{~min}^{-1}\right)$ at a heating rate of $10^{\circ} \mathrm{C} \mathrm{min}-1$, using a Perkin Elmer TAC 7/DX controller with a TGA 7 furnace operating at temperatures between 30 and $500^{\circ} \mathrm{C}$.

Disk sections of approximately $10 \mathrm{mg}$ mass were removed from electrolyte films and transferred to $40 \mu \mathrm{L}$ aluminium DSC cans which were sealed within the preparative glovebox. The purge gas used in all the analyses was high purity argon supplied at a constant $35 \mathrm{~cm}^{3} \mathrm{~min}^{-1}$ flow rate. Thermal analysis was carried out with a Mettler TC11 controller and a DSC 20 oven equipped with a cooling accessory. All samples were subjected to a $5{ }^{\circ} \mathrm{C} \mathrm{min}^{-1}$ heating rate and were characterised between -40 and $200^{\circ} \mathrm{C}$.

XRD analysis was carried out on milled homogeneous polymer/salt mixtures packed into $0.7 \mathrm{~mm}$ diameter Lindemann tubes under an argon atmosphere. The samples were annealed at $80^{\circ} \mathrm{C}$ for several hours to dissolve and distribute the salt in the polymer host. The Lindemann tubes were sealed with quick-setting epoxy resin and analysed with a Stoe STADI/P high resolution diffractometer equipped with a germanium monochromator operating in transmission mode. $\mathrm{Cu} \mathrm{K}_{\alpha}$ radiation was used throughout and the data was collected using a small angle linear position sensitive detector

\section{Results and Discussion}

Conductivity measurements In PEO-based electrolyte systems the change of gradient in linear log (conductivity) versus $1 / \mathrm{T}$ graphs at temperatures close to $60^{\circ} \mathrm{C}$ is normally associated with the fusion of uncomplexed polymer spherulites. In many systems the variation of the electrolyte conductivity with temperature helps to locate the electrolyte composition being studied on the polymer-rich side of the system eutectic composition. Generally the almost completely amorphous electrolyte samples found near the system eutectic, or in the salt-rich region of the system phase diagram, show non-linear log (conductivity) versus $1 / \mathrm{T}$ plots. The conductivity data of the zinc triflate electrolyte compositions with $\mathrm{n}$ close to or below 20, show non-linear variation of conductivity with temperature, suggesting that in this composition range the electrolytes are essentially amorphous. The variation of ionic conductivity with temperature observed in the $\mathrm{PEO}_{\mathrm{n}}$ $\mathrm{Zn}$ triflate electrolyte system was, in this respect, similar to that reported for other zinc salts and the majority of mono- and multivalent cation PEO-based electrolytes which also show amorphous morphology in this region of high salt concentration.

The total ionic conductivity of the zinc triflate electrolte was found to vary little with sample composition over almost the whole range of electrolyte compositions studied. The results shown in Figure 1 confirm the presence of a maximum at a EO/Zn ratio of about 60 . This aspect of the behaviour of polymer electrolytes is quite different from aqueous or non-aqueous liquid electrolyte systems which show an increase in conductivity as the salt concentration is raised and the numbe of charge carriers increases. In conventional electrolytes further increase in salt content results in a reduction of conductivity as ion-ion effects begin to arise and impede ion transport. Where the "solvent" is a polymer, low salt concentrations allow the growth of spherulites and the guest salt species accumulate in the inter-spherulitic amorphous regions of the electrolyte volume. The total ionic conductivity in this region is normally fairly low as the presence of polymer spherulites convolutes the conduction pathways, restricted to the amorphous phase.

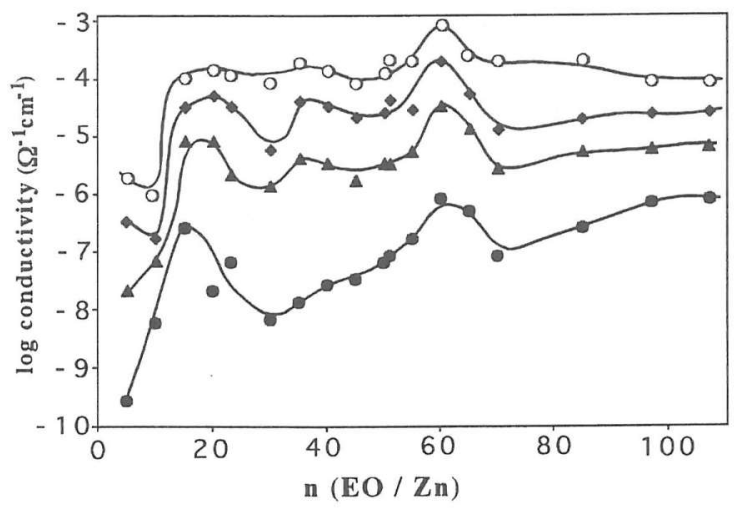

Fig. 1. conductivity isotherms for electrolyte compositions of $\mathrm{n}$ between 5 and $100\left(30^{\circ} \mathrm{C}, 40^{\circ} \mathrm{C}, 50^{\circ} \mathrm{C}, \mathrm{O} 80^{\circ} \mathrm{C}\right)$

The region of composition of $\mathrm{n}$ between about 15 and 30 is of particular interest. While at low temperatures a significant variation of conductivity with composition is evident, at higher temperatures this becomes very much less marked. The location of the eutectic composition of the zinc triflate - polymer system between $\mathrm{n}=15$ and 30 would explain a conductivity enhancement as 
clectrolytes within this region would complete their transformation to the liquid phase at lower temperatures than compositions further from the eutectic composition. The observed enhancement would not be expected at temperatures significantly above the eutectic temperature, because in this temperature range the electrolytes are amorphous over the entire range of compositions studied ( $\mathrm{n}$ between 5 and 100).

The conductivity behaviour of this electrolytic medium is further complicated by the low dielectric constant of the amorphous polymer component. The presence of ion aggregrates has been confirmed in multivalent systems $[11,19,20]$ and at moderate and high salt concentration the association of ions into pairs or aggregates reduces the number of species which can contribute to the total conductivity and therefore impedes charge transfer. This effect may be one of the causes of the observed reduction in total ionic conductivity observed in the PEO-zinc triflate system at values of $\mathrm{n}$ between 20 and 5 . Clearly the ionic conductivity in polymer electrolytes is a complex function of the morphology of the environment, the state of aggregation of the species present and the presence of water or solvent residues which may enhance the conductivity by introducing more free volume into the polymer host or conversely impede ion transport by inhibition of complexation sites.

Thermal analysis The results of thermal analysis of electrolyte samples, illustrated in Figure 2, confirmed the observations reported with conductivity and X-ray experiments. At low concentrations of guest salt the morphology of the samples is largely crystalline and the uncomplexed polymer forms clearly-defined spherulites. The crystalline polymer melts with a clearly observable endotherm at close to $60^{\circ} \mathrm{C}$. As the amount of salt is increased the morphology

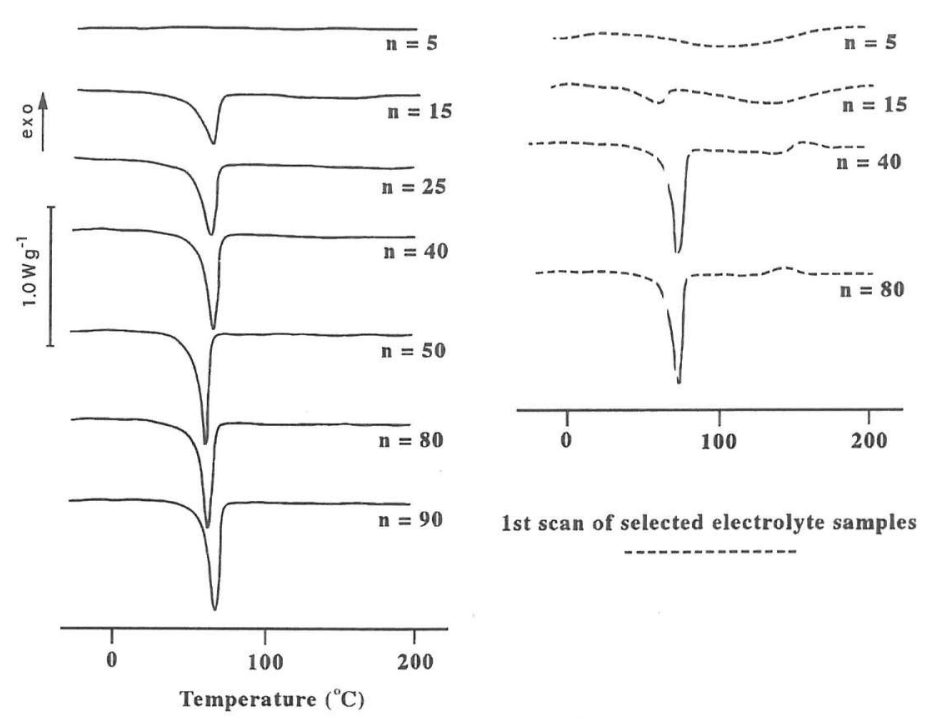

Fig. 2. DSC behaviour of selected electrolyte compositions of the electrolyte is progressively changed to an almost entirely amorphous structure and the kinetics of the spherulite growth are slowed by interactions between the heteroatoms in the polymer structure and the salt. In the first scan carried out on samples with high salt concentrations a broad endotherm was observed between 80 and $180^{\circ} \mathrm{C}$. This may be explained by the existence of a poorly defined salt-polymer complex. Broad peaks with similar characteristics have been observed with several other systems, unfortunately in this composition range the samples were too amorphous to be characterised by X-ray techniques. These results suggest that the electrolyte system formed by poly(ethylene oxide) and zinc triflate probably forms a salt/polymer complex in the salt-rich region of composition ( $\mathrm{n}$ close to 5) and a eutectic at $\mathrm{n}$ between 10 and 20 . Thermal decomposition of the host polymer was clearly visible at higher temperatures where characteristic irregular exotherms were observed.

At certain electrolyte compositions poorly defined exotherms were registered in the analysis of the first scan of electrolyte samples. These exotherms were not present in the second scan and lead to the suggestion that limited oxidative decomposition may occur during the first exposure of the sample to moderate temperatures. Exotherms of this nature are often of such weak intensity that they are ignored, however in the first scans of electrolytes with composition $n=40$ and 80 these peaks are readily identifiable. Clearly this aspect of the thermal behaviour of the commercial host polymer, which also contains a mixture of process residues, anti-oxidants and thermal stabilisers, requires further characterisation.

The thermal decomposition of electrolyte samples was studied by TGA. The salt destabilises the polymer network and decomposition occurs by a multi-stage process which has an onset at about $290^{\circ} \mathrm{C}$ in some compositions (Figure 3). The precise temperature at which the process begins is determined by the electrolyte composition. As in previously characterised systems [22, 23], the electrolytes with higher salt concentrations were found to be thermally less stable.

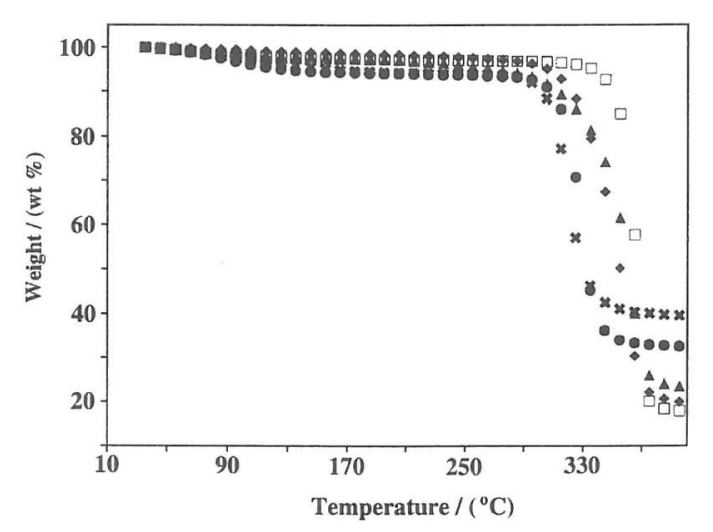

Fig. 3. Thermogravimetric curves of $\mathrm{PEO}_{n} \mathrm{Zn}\left(\mathrm{CF}_{3} \mathrm{SO}_{3}\right)_{2}$ $(\mathrm{n}=80 \square, 65 \diamond, 55 \mathbf{\Delta}, 20 \bullet$ and $15 *)$ 
$\mathrm{X}$-ray analysis The data from X-ray structure analysis, illustrated in Figure 4, confirms the presence of polymer spherulites in electrolytes with low salt concentrations and the expected increase in crystalline morphology as the amount of guest salt is decreased. No evidence of crystalline complexes between the polymer and the salt was found however. Although relatively few polymer electrolyte systems have been characterised using X-ray techniques, in most of these studies difficulties have been encountered in obtaining clear structural information within the saltrich region of composition and in several multivalent cation-based systems $[13,16,21]$ this region has been found to be essentially amorphous. This problem was also apparent in the zinc triflatebased polymer electrolyte and the predominantly amorphous nature of the environment masked relevant structural details.

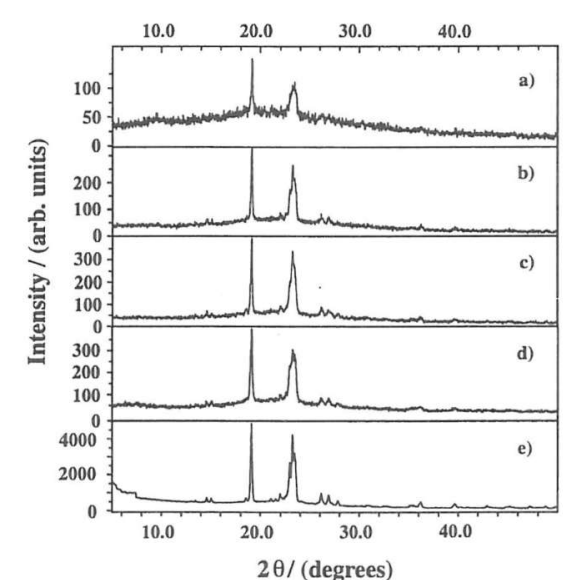

Fig. 4. X-ray diffraction spectra of pure polymer and electrolytes of $n$ between 70 and 15 (a) 15, b) 40 , c) 60 , d) 70 and e) pure polymer.

\section{Conclusions}

The semi-crystalline electrolytes formed by solvent casting mixtures of zinc triflate and commercial poly(ethylene oxide) shows moderate ionic conductivity over a wide range of compositions. Some evidence was obtained from DSC studies which supports the proposal that a crystalline salt/polymer complex is formed in this divalent salt system at compositions within the high salt concentration region, however the existence of this complex was not confirmed by X-ray techniques. The difficulty in obtaining structural proof for the formation of this complex probably originates in the slow crystallisation kinetics observed in this system within the salt-rich composition range. Samples for X-ray analysis could not be prepared by the more convenient solvent casting technique due to the need to avoid exposure of the sample to water during structural data collection. Sealed Lindemann tubes were used to protect samples from atmospheric humidity and electrolyte compositions were prepared for structural study by annealing mixtures of electrolyte. This restriction may also have contributed to the difficulty in obtaining conclusive evidence for the formation of a salt-polymer complex. Thermal stability of electrolytes produced in the range of composition of $\mathrm{n}$ between 5 and 120 was similar to other previously characterised systems based on the same commercial host polymer.

References

1. M. B. Armand, J. M. Chabagno and M. Duclot, in "Second International Meeting on Solid Electrolytes", St. Andrews, Scotland, 20-22 Sept., 1978, Extended Abstract.

2. P. G. Bruce and C. A. Vincent, J. Chem. Soc., Faraday Transactions, 89 (17) (1993) 3187.

3. J. R. MacCallum and C. A. Vincent, eds, "Polymer Electrolyte Reviews", Vol 1 \& 2, Elsevier Applied Science, London and New York, 1987 and 1989.

4. F. M. Gray, "Solid Polymer Electrolytes - fundamentals and technological applications", VCH Publishers, Inc., New York, 1991.

5. A. Patrick, M. Glasse, R. Latham and R. Linford, Solid State Ionics, $18 / 19$ (1986) 1063.

6. M. D. Sheldon, M. D. Glasse, R. J. Latham and R. G. Linford, Solid State Ionics, 34 (1989) 135.

7. H. Yang, R. Huq and G. C. Farrington, Solid State Ionics 40/41 (1990) 663.

8. M. D. Glasse, R. G. Linford and W. S. Schlindwein, p. 203; V. C. Z. Bermudez, J. Morgado, T. M. A. Abrantes and L. Alcácer, p. 251; H. Yang and G. C. Farrington, p. 265; in "Proc. 2nd Intern. Symp. on Polymer Electrolytes", ed. B. Scrosati, Elsevier Applied Science, London, 1990.

9. M. D. Glasse, R. J. Latham, R. G. Linford and R. A. J. Pynenburg, Solid State Ionics, 53-56 (1992) 1111.

10. J. M. G. Cowie, A. T. Anderson, M. Andrei, A. C. S. Martin and C. Roberts, Electrochimica Acta, 37 (1992) 1539.

11. A. Wendsjo, J. Lindgren, J. O. Thomas and G. C. Farrington, Solid State Ionics, $53-56$ (1992) 1077 .

12. M. Giua, S. Panero, B. Scrosati, X. Cao and S. G. Greenbaum, Solid State Ionics, 83 (1996) 73 13. G. C. Farrington and R. G. Linford, in "Polymer Electrolyte Reviews", Vol 1 \& 2, eds., J. R. MacCallum and C. A. Vincent, Elsevier Applied Science, London and New York, 1987 and 1989 p. 255 .

14. M. J. Smith and C. J. R. Silva, Solid State Ionics, 58 (1992) 269.

15. C. D. Robitaille and D. Fauteux, J. Electrochem. Soc., 133 (1986) 315.

16. M. A. Mehta, P. Lightfoot and P. G. Bruce, Chem. Mater., 5 (1993) 1338

17. A. Wendsjo and H. Yang, in "Proc. 2nd Intern. Symp. on Polymer Electrolytes", ed. B. Scrosati, Elsevier Applied Science, London, 1990 p. 225.

18. M. J. Smith and C. J. R. Silva, Electrochimica Acta, 40 (1995) 2389.

19. A. Wendsjö, J. Lindgren and C. Paluszkiewicz, Electrochimica Acta, 37 (1992) 1689.

20. H. S. Choe and G. C. Farrington, Solid State Ionics, 57 (1992) 35.

21. J. J. Cruz Pinto, M. M. Silva, M. J. Smith and C. J. R. Silva, J. Thermal Analysis, 40 (1993) 641.

22. G. K. Jones, G. C. Farrington and A. R. McGhie, in "Proc. 2nd Intern. Symp. on Polymer Electrolytes", ed. B. Scrosati, Elsevier Applied Science, London, 1990, p. 239.

23. G. G. Cameron, M. D. Ingram, M. Younus Qureshi, H. M. Gearing, L. Costa and G. Camino, Eur. Polym. J., 25 (1989) 779. 